\title{
Studies on the Nephrotoxicity of Aminoglycoside Antibiotics and Protection from These Effects (6): A Mechanism for the Suppressive Action of Latamoxef on Intrarenal Tobramycin Level
}

\author{
Ryoji KOJIMA, Mikio ITO and Yoshio SUZUKI \\ Department of Pharmacology. Faculty of Pharmacy, Meijo University. \\ 15 Yagoto Urayama, Tenpaku-ku, Nagoya 468, Japan \\ Accepted February 2. 1990
}

\begin{abstract}
The mechanism by which latamoxef ( $\mathrm{MOX}$ ) reduces intrarenal tobramycin (TOB) levels was studied. When TOB (90 mg/ $\mathrm{kg} /$ day, s.c.) and LMOX (500 or 2000 $\mathrm{mg} / \mathrm{kg} / \mathrm{day}, \mathrm{s.c}$.) were given simultaneously at separate sites to rats, 20 to $30 \%$ reductions in intrarenal TOB concentration were found on the 1 st and 3 rd days, as compared with the results from using TOB alone. The treatment with the reaction mixture of TOB and LMOX, which was preincubated for $3 \mathrm{hr}$ at $37^{\circ} \mathrm{C}$ to form the complex of TOB and $L M O X$, resulted in a greater suppression of TOB level in the kidney than administration of TOB and LMOX concomitantly at separate sites. When LMOX was given to rats 0.5 or $1.5 \mathrm{hr}$ before s.c. injection of TOB, there were significant reductions in intrarenal TOB concentration. However, treatment with LMOX 5 hr before, as well as 1.5 or $5 \mathrm{hr}$ after TOB injection, was ineffective in reducing the accumulation of TOB in the kidney. In the rats given both drugs simultaneously. the urinary excretion pattern of TOB almost overlapped with that of LMOX. Additionally, we detected a complex of TOB and LMOX in the urine of rats given both drugs simultaneously. These results suggest that the suppressive action of LMOX on intrarenal TOB level is due to the interaction of TOB and LMOX.
\end{abstract}

We previously reported that tobramycin (TOB)-induced nephrotoxicity in rats is prevented by combining TOB with latamoxef (LMOX) and that the protection induced by LMOX is due to reduced intrarenal TOB concentration (1). It has been proposed that aminoglycoside antibiotics (AGs) bind to brush border membranes (BBMs), which have been shown to be the binding site for AGs in renal proximal tubular cells (2), and then are transported and accumulate in lysosomes (3). Sastrasinh et al. (2) have suggested that an interaction of $A G s$ with BBMs is due to the electrostatic force between positive and negative charges of AGs and acidic phospholipids of BBMs, including phosphatidylinositol, respectively. Other investigators have also shown that polycationic AGs bind negatively charged phospholipids $(4-6)$.

LMOX has two carboxyl groups in the molecule and thereby is negatively charged at physiological $\mathrm{pH}$. Therefore, to clarify the mechanism by which LMOX reduces the intrarenal TOB level, we have hypothesized that positively charged TOB may be able to bind to negatively charged LMOX, that the molecular interaction between TOB and LMOX may inhibit the binding of TOB to BBMs, and that the inhibition of TOB binding to BBMs may lead to the decrease in TOB level in the kidney.

Recently, we have demonstrated by paper electrophoresis, thin layer chromatography (TLC), and ultraviolet and infrared spectrophotometries that $\mathrm{TOB}$ directly interacts with LMOX in vitro (7). In addition, we have shown that the interaction of TOB and LMOX is involved in the inhibition by LMOX of TOB binding to BBMs isolated from rat kidney cortex in vitro (8). Therefore, in the present study, to clarify whether the interaction between TOB and LMOX in Vivo associated with the suppressive action of LMOX on in- 
trarenal TOB level, we investigated the effect of a reaction mixture of $T O B$ and $L M O X$. which was preincubated for $3 \mathrm{hr}$ at $37^{\circ} \mathrm{C}$ to form a complex of TOB and LMOX, on TOB level in the kidney, and the effect on intrarenal TOB concentration of LMOX administered before or after TOB injection. Additionally, the time courses of urinary excretion of TOB and LMOX after single s.c. injection of TOB alone. LMOX alone or of both drugs, and the change in intrarenal TOB level at various times after the administration of TOB were determined. Furthermore, we attempted to detect a complex of TOB and LMOX in the urine.

\section{Materials and Methods}

Animals: Male Sprague-Dawley rats weighing $230 \mathrm{~g}$ (Japan SLC, Shizuoka, Japan) were used in all experiments. These animals were housed in an airconditioned room at $24 \pm 1^{\circ} \mathrm{C}$

Drugs: Drugs used were tobramycin (TOB, Shionogi Co., Ltd.) and latamoxef sodium (LMOX, Shionogi Co., Ltd.). Both TOB and LMOX were dissolved in saline.

Drug treatment: 1) Effect of the reaction mixture of TOB and LMOX on intrarenal TOB concentration: Rats were given a daily s.c. dose of TOB $(90 \mathrm{mg} / \mathrm{kg})$ for 3 consecutive days. To correct for the effect of sodium intake in the LMOX injection on intrarenal TOB concentration, subcutaneous injection of a $\mathrm{NaCl}$ solution was also carried out when TOB was given alone. In other groups, TOB $(90 \mathrm{mg} / \mathrm{kg} /$ day, s.c.) and LMOX at 500 or $2000 \mathrm{mg} / \mathrm{kg} /$ day (which are s.c. doses to prevent TOBinduced nephrotoxicity (1)) were administered simultaneously at separate sites on the rats for 3 days. In addition, the solutions of TOB $(90 \mathrm{mg} / \mathrm{kg}$ ) and LMOX (500 or 2000 $\mathrm{mg} / \mathrm{kg}$ ) dissolved separately in saline were mixed and incubated for $3 \mathrm{hr}$ at $37^{\circ} \mathrm{C}$ to form a complex of both drugs (7). The resultant reaction mixture was given subcutaneously to rats for 3 days. The intrarenal TOB concentrations were measured on the $1 \mathrm{st}$ and $3 \mathrm{rd}$ days.

2) Effect of LMOX administered at a different time from TOB injection on intrarenal TOB concentration: LMOX ( $500 \mathrm{mg} / \mathrm{kg}, \mathrm{s.c}$.) was given at a different site on the rats from
TOB injection $0.5,1.5$ or $5 \mathrm{hr}$ before, and 1.5 or $5 \mathrm{hr}$ after TOB (90 mg/kg. s.c.) injection. The intrarenal TOB concentration was measured $24 \mathrm{hr}$ after the injection of TOB. Also, the effect of simultaneous treatment with both drugs on intrarenal TOB level was evaluated.

Measurement of intrarenal TOB concentration: The intrarenal TOB concentration was measured by the previously reported method (1), except for centrifugation of the kidney homogenate at $9.000 \times \mathrm{g}$ for $15 \mathrm{~min}$ and the measurement of TOB concentration by substrate-labeled fluorescent immunoassay (SLFIA. Ames TDA tobramycin assay kit, Miles-Sankyo Co. Ltd.. Tokyo. Japan) (9).

Time courses of urinary excretion of TOB and LMOX: Ureteral catheterization and urine collections were carried out by slightly modyfying the methods of Stizer and MartinezMaldonado (10) and Cojocel et al. (11) The rats given $\mathrm{H}_{2} \mathrm{O}(8 \mathrm{ml}$, p.o.) were anesthetized with sodium pentobarbital (32.4 mg/kg, i.p.), placed on a temperature-regulated hot plate. and subjected to tracheostomy. The left kidney was exposed by midline abdominal incision, and the left ureter was catheterized with polyethylene tubing (PE-10) for urine collection. During animal preparation, the fluid loss was replaced by an intravenous infusion of saline. After an equilibration period of $30 \mathrm{~min}$, the rats were given subcutaneously TOB (90 mg/ $\mathrm{kg}$ ) alone. LMOX (500 mg/ $\mathrm{kg}$ ) alone or TOB $(90 \mathrm{mg} / \mathrm{kg})$ and LMOX (500 $\mathrm{mg} / \mathrm{kg}$ ) concomitantly at separate sites. Urine samples were collected in bottles through a ureteral catheter for periods of 30 or $60 \mathrm{~min}$ each until $8 \mathrm{hr}$ after drug administration. TOB concentrations in the urine sampled at times after TOB injection were measured by SLFIA. LMOX in the urine was separated by TLC as previously reported (7); the spots of LMOX on a thin layer plate were scanned with a densitometer (S-910. Shimadzu. Japan) with a zig-zag scanning system to quantify the amounts of LMOX; and urinary concentrations of LMOX were calculated from the standard curve of $L M O X$ prepared by TLC and densitometry.

Time course of intrarenal TOB concentration: After subcutaneous injection of TOB $(90 \mathrm{mg} / \mathrm{kg})$, the TOB concentrations in the left kidney at various time intervals were 
measured as described above.

Detection of a complex of TOB and LMOX in urine: Under anesthesia with pentabarbital, the rats fitted with a ureteral catheter as previously described were given TOB (90 mg/ $\mathrm{kg}$. s.c.) alone. $\operatorname{LMOX}(500 \mathrm{mg} / \mathrm{kg}$. s.c.) alone or both drugs simultaneously. In other rats, LMOX was injected $5 \mathrm{hr}$ before or after TOB injection. At various times after drug injection. $1 \mu \mid$ of urine was obtained directly from the ureteral catheter, but not the collection bottle. to avoid sampling any complexes of TOB and LMOX formed within the bottle during the urine collection and was subjected to cellulose acetate electrophoresis. The cellulose acetate electrophoresis of the urine was performed for $1 \mathrm{hr}$ with $70 \mathrm{mM}$ Tris- $\mathrm{HCl}$ buffer ( $\mathrm{pH} \mathrm{7.4),} \mathrm{and} \mathrm{then} \mathrm{the} \mathrm{cellulose} \mathrm{acetate} \mathrm{mem-}$ brane $(7 \times 10 \mathrm{~mm})$ was placed on an agar (Pearlcore, Eiken, Japan) plate containing about $1 \times 10^{3} \quad \mathrm{CFU} / \mathrm{ml}$ of Pseudomonas aeruginosa SR-24 (Shionogi Co.. Ltd., Japan). After the incubation of the plate for $18 \mathrm{hr}$ at $37^{\circ} \mathrm{C}$, the inhibition zone of the bacterial growth caused by TOB or LMOX was observed. Additionally, aliquots of a mixture of TOB $(30 \mathrm{mM})$ and LMOX (210 $\mathrm{mM}$ ) incubated at $37^{\circ} \mathrm{C}$ for $30 \mathrm{~min}$ in vitro were analyzed by cellulose acetate electrophoresis and the microbiological test as described above.

Statistical analysis: The data were subjected to one-way analysis of variance and the Duncan multiple range test, or non-parametric statistics. The data in the text, figures and table are expressed as the mean \pm S.D. Differences were considered significant if the $P$ value was $<0.05$.

\section{Results}

1. Effect of the reaction mixture of TOB and LMOX on intrarenal TOB concentrations: As shown in Fig. 1, the intrarenal TOB level in the rats receiving TOB alone was $254.9 \pm 52.1$ and $488.3 \pm 24.5 \mathrm{~kg} / \mathrm{g}$ tissue wet weight on the 1 st and 3 rd days, respectively. Simultaneous treatment with TOB and LMOX (500 $\mathrm{mg} / \mathrm{kg}$ ) at separate sites on the rats reduced the intrarenal TOB concentration by $28 \%$ $(184.7 \pm 26.6 \mathrm{~kg} / \mathrm{g}$ tissue wet weight) and
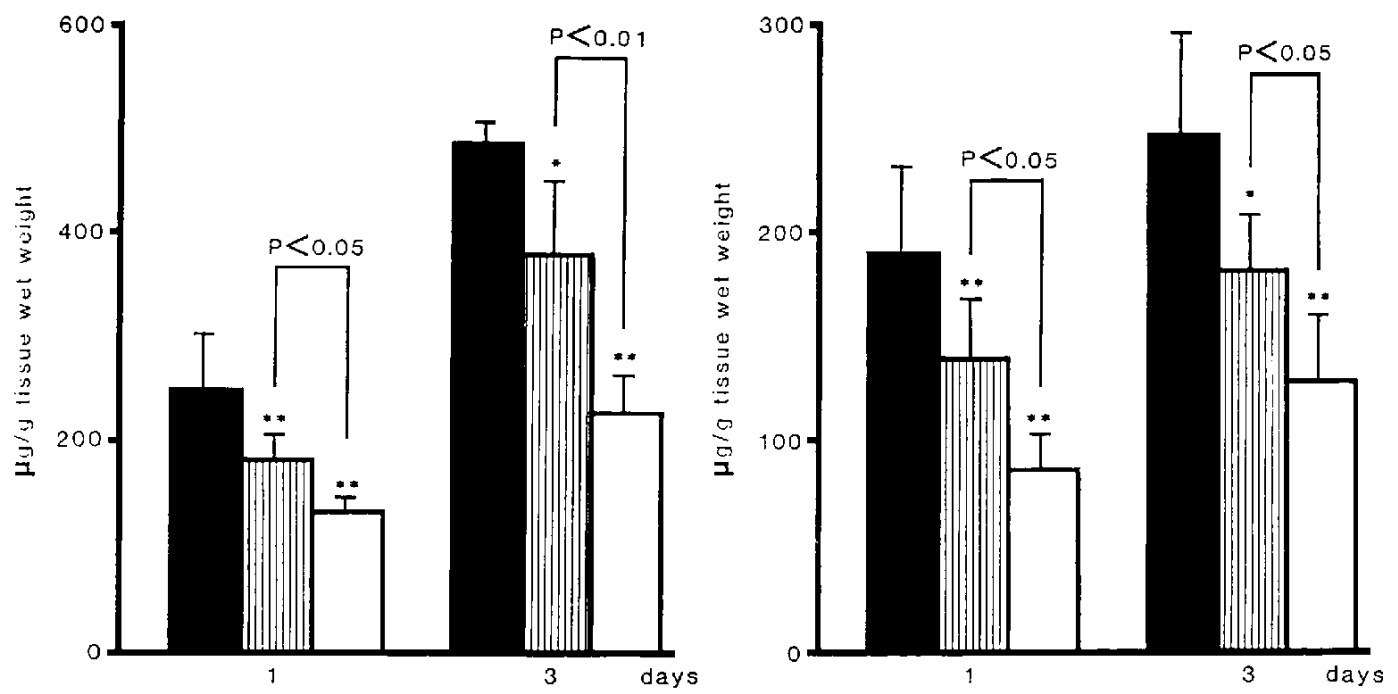

Fig. 1. Effect of the reaction mixture of TOB and LMOX on intrarenal TOB concentration. TOB. $90 \mathrm{mg} / \mathrm{kg}$ : (III TII ) : TOB, $90 \mathrm{mg} / \mathrm{kg}+$ LMOX, 500 or $2000 \mathrm{mg} / \mathrm{kg}$, at separate sites: ( $\square$ ): TOB, $90 \mathrm{mg} / \mathrm{kg}+\mathrm{LMOX} .500$ or $2000 \mathrm{mg} / \mathrm{kg}$, preincubated reaction mixture. TOB alone, LMOX alone or the mixture of $T O B$ and $L M O X$ was incubated for $3 \mathrm{hr}$ at $37^{\circ} \mathrm{C}$ and then administered to the rats. The values are expressed as the mean $\pm S$. D. from 7 rats. $\because P<0.05,{ }^{* *}: P<0.01$, compared to TOB alone. 
$22 \%$ (381.4 $471.8 / \mathrm{gg} / \mathrm{g}$ tissue wet weight) by the 1 st and 3rd days, respectively. When the reaction mixture of TOB and LMOX was given to rats, the intrarenal TOB level was lower $(134.7 \pm 17.8$ and $226.5 \pm 41.1 \mu \mathrm{g} / \mathrm{g}$ tissue wet weight on the 1 st and 3 rd days, respectively) than that observed when TOB and LMOX were given simultaneously at separate sites. Likewise, in the rats receiving a combination with LMOX $(2000 \mathrm{mg} / \mathrm{kg})$, the ad ministration of the preincubated reaction mixture resulted in a greater suppression of intrarenal $T O B$ accumulation than that of $T O B$ and LMOX at different sites.

2. Effect of LMOX administered at a different time from TOB injection, on intrarenal TOB concentration: As shown in Fig. 2, the administration of $L M O X$ at 0.5 or $1.5 \mathrm{hr}$ before TOB injection reduced the intrarenal TOB concentration to a level similar to that observed in simultaneous treatment with both drugs at different sites. However, when LMOX was administered $5 \mathrm{hr}$ before, and 1.5 or $5 \mathrm{hr}$ after TOB injection, no significant differences in intrarenal TOB level were observed. In addition, the renal TOB levels of rats treated with LMOX at 1.5 or $5 \mathrm{hr}$ after TOB injection seemed to be less, but not significantly so. than those of rats given LMOX $5 \mathrm{hr}$ before TOB injection.

3. Time courses of urinary excretion of
TOB and LMOX: Figure 3 illustrates the time course of urinary TOB and $L M O X$ levels after single s.c. injection of TOB or $L M O X$. The urinary level of $T O B$ in rats receiving $T O B$ alone peaked at around $1 \mathrm{hr}$ after the drug administration and then gradually decreased untif $8 \mathrm{hr}$ after TOB injection (Fig. 3A). The excretion level of LMOX in the urine also peaked at about $1 \mathrm{hr}$ after the injection of LMOX (Fig. 3B). Additionally, in rats given both drugs simultaneously at different sites, the urinary excretion pattern of TOB almost overlapped with that of LMOX, and urinary excretion levels of TOB and LMOX showed their peaks at the same time (Fig. $3 \mathrm{C}$ ). In the rats given both drugs, regardless of a significant reduction in the intrarenal TOB level, urinary $T O B$ levels were not higher than those in the rats treated with TOB alone (Fig. 3C). This may result from an interference in the measurement of TOB by the formation of a complex of TOB and LMOX in the bottle during the urine collection for 30 or 60 min.

4. Time course of intrarenal TOB concentration: After single s.c. injection of $T O B$, the intrarenal TOB level rapidly increased, up to a level of $170.7 \pm 25.2 \mathrm{~kg} / \mathrm{g}$ tissue wet weight at $2 \mathrm{hr}$, and then gradually increased to a concentration of $221.1 \pm 20.1 \mathrm{~kg} / \mathrm{g}$ tissue wet weight at $8 \mathrm{hr}$ (Table 1).

5. Detection of a complex of TOB and

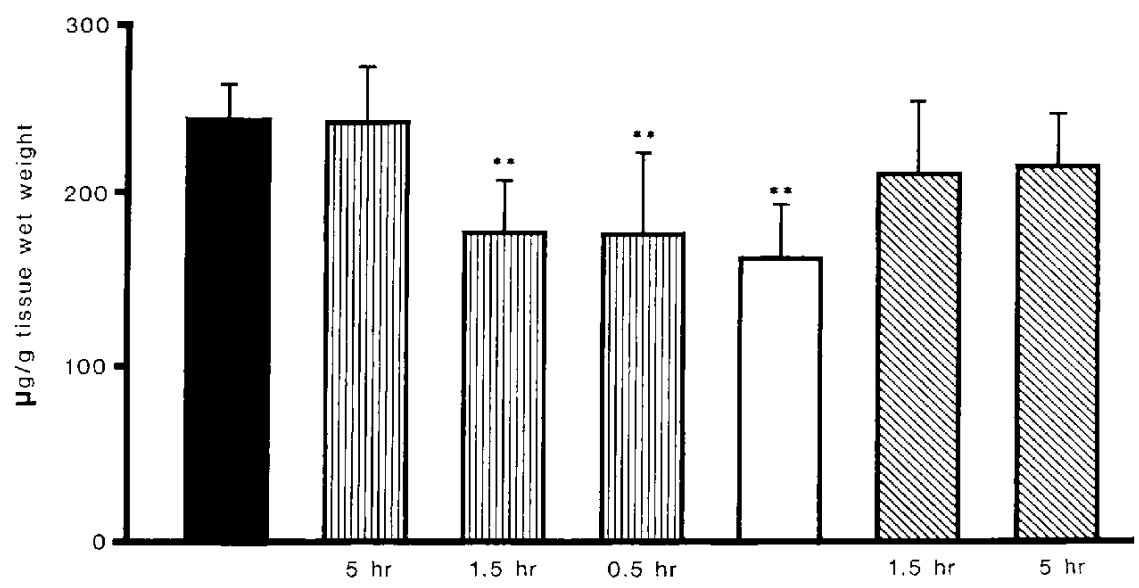

Fig. 2. Effect of LMOX administered at a different time from TOB injection on intrarenal TOB concen-

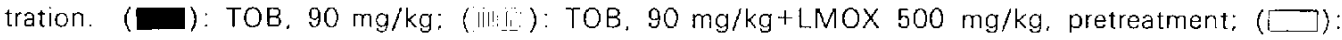
TOB, $90 \mathrm{mg} / \mathrm{kg}+\mathrm{LMOX}, 500 \mathrm{mg} / \mathrm{kg}$, simultaneous treatment: (GWM ): TOB, $90 \mathrm{mg} / \mathrm{kg}+\mathrm{LMOX}, 500$ $\mathrm{mg} / \mathrm{kg}$, posttreatment. The values are expressed as the mean \pm S.D. from 7 rats. **:P<0.01, compared to TOB alone. 
A

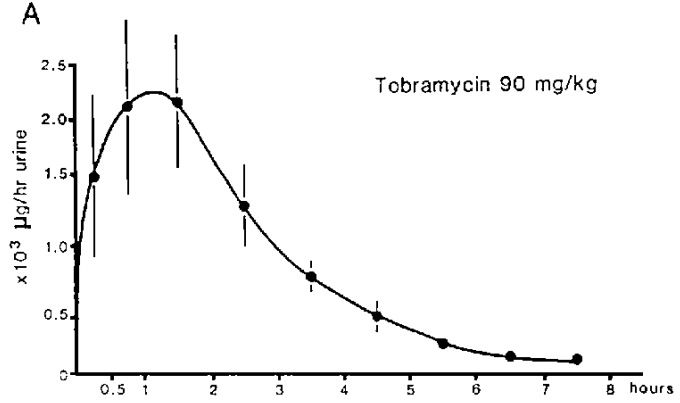

B

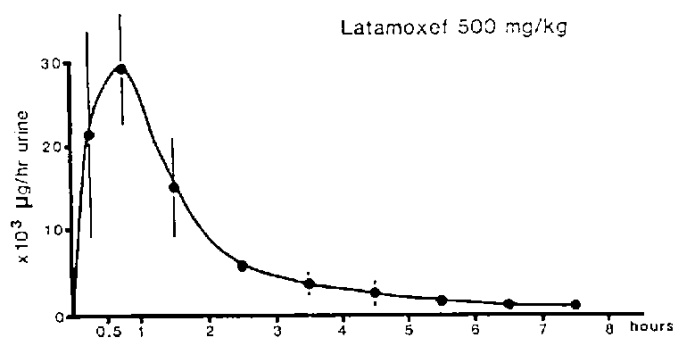

C

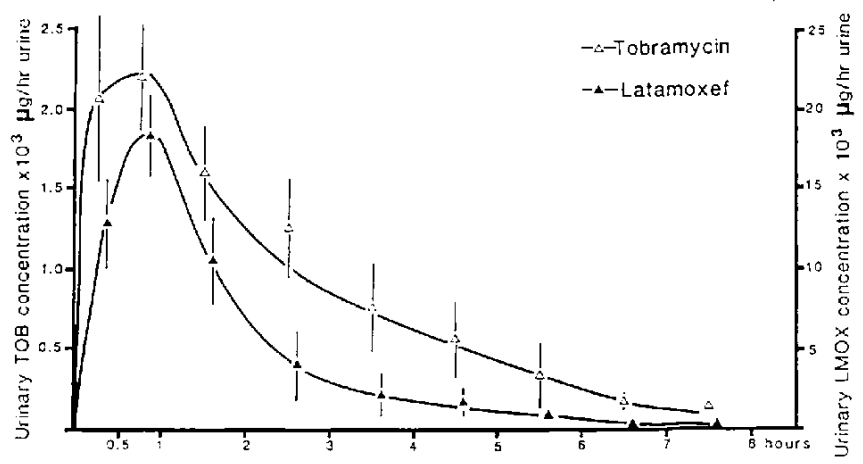

Fig. 3. Time courses of urinary $T O B$ and $L M O X$ concentration after subcutaneous injection of $T O B$ and LMOX. A: TOB, $90 \mathrm{mg} / \mathrm{kg}$ alone: B: LMOX, $500 \mathrm{mg} / \mathrm{kg}$ alone: C: TOB. $90 \mathrm{mg} / \mathrm{kg}+\mathrm{LMOX}, 500 \mathrm{mg} / \mathrm{kg}$. The values are expressed as the mean \pm S.D. from 8 rats.

Table 1. Time course of intrarenal TOB concentration after single s.c. injection of TOB

\begin{tabular}{|c|c|c|c|c|c|c|}
\hline & \multicolumn{6}{|c|}{ Time $(\mathrm{hr})$} \\
\hline \multirow{2}{*}{-} & 0.5 & 1 & 2 & 3 & 5 & 8 \\
\hline & \multicolumn{6}{|c|}{$\mu \mathrm{g} / \mathrm{g}$ tissue wet weight } \\
\hline TOB, $90 \mathrm{mg} / \mathrm{kg}$ & $\begin{array}{l}115.54 \\
\pm 16.84\end{array}$ & $\begin{array}{l}163.33 \\
\pm 21.60\end{array}$ & $\begin{array}{l}170.74 \\
\pm 25.21\end{array}$ & $\begin{array}{l}195.55 \\
\pm 22.04\end{array}$ & $\begin{array}{l}201.80 \\
\pm 47.25\end{array}$ & $\begin{array}{l}221.11 \\
\pm 20.12\end{array}$ \\
\hline
\end{tabular}

The values are expressed as the mean \pm S.D. from 7 rats

LMOX in urine: Figure 4 illustrates the interaction of TOB and LMOX in vitro analyzed by cellulose acetate electrophoresis and the microbiological test. The inhibition zone of cell growth caused by TOB alone was observed as a single zone on the cathode side, and the zone for LMOX alone was also observed as a single zone on the anode side. No significant changes in the inhibition zone were observed when TOB and LMOX were spotted separately at the origin of the cellulose acetate membrane. However, the analysis of the mixture of both compounds showed that at different sites from TOB alone on the cathode side, a new inhibitory zone appeared (Fig. 4). Additionally, the densitometric pattern of LMOX on the cellulose acetate membrane indicated that the peaks of absorption of LMOX $(\lambda=268 \mathrm{~nm})$ were observed on the cathode side as well as on the anode side, and that the peak on the cathode side overlapped with the newly found inhibition zone (data not shown). This result indicated that the newly found inhibition zone was a manifestation of a complex of TOB and LMOX. When the urine of the rat given both drugs simul- 


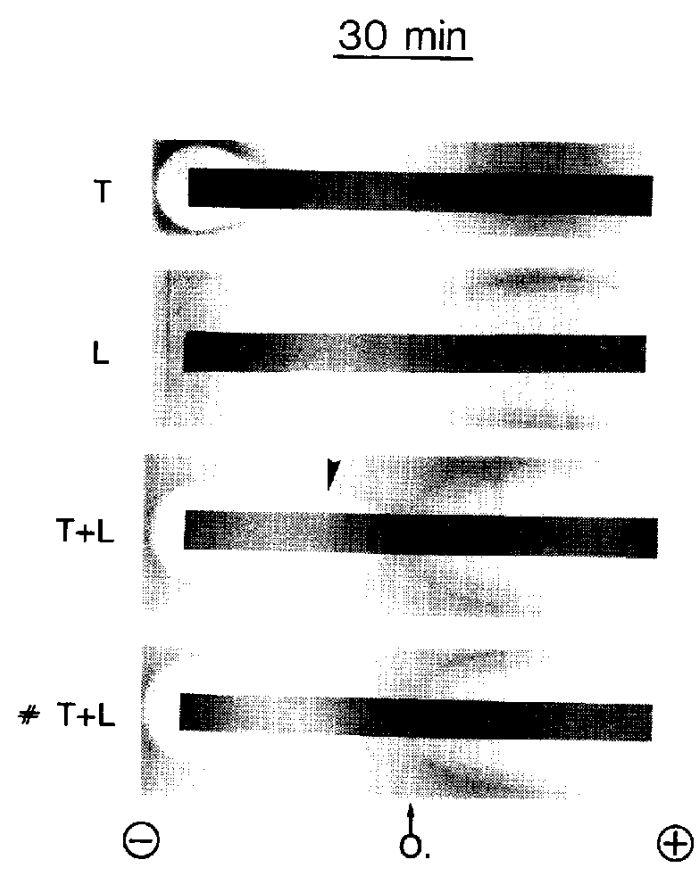

Fig. 4. Detection of the complex of TOB and LMOX in vitro. Aliquots of the mixture of TOB $(30 \mathrm{mM})$ and $L M O X(210 \mathrm{mM})$ incubated at $37^{\circ} \mathrm{C}$ for $30 \mathrm{~min}$ in vitro were analyzed by the method described in Materials and Methods. O.: origin: T: TOB alone; L: $L M O X$ alone: $T+L$ : reaction mixture of $T O B$ and LMOX: $\$ T+L$ : TOB and LMOX (the drugs were separately spotted at the origin). Arrow head represents the complex of both drugs.

taneously was analyzed, a new inhibition zone was clearly observed on the cathode side, as shown in the in vitro study (Fig. 5). In addition, the new inhibition zone was undetectable when the urine containing each drug was spotted separately on the cellulose acetate membrane, indicating that the new inhibition zone was not due to an artifact produced by the experimental procedure. Additionally, no new inhibition zones were detected in the urine obtained from rats receiving $L M O X$ at $5 \mathrm{hr}$ before or after TOB injection (Fig. 6).

\section{Dișcussion}

The objective of the present study was to examine whether the interaction of TOB and LMOX in vivo is involved in the suppressive action of LMOX on intrarenal TOB level.
In the first set of experiments, we found that the treatment of rats with the reaction mixture of TOB and $L M O X$ resulted in a greater reduction of intrarenal TOB level than that with TOB and LMOX at separate sites (Fig. 1). Analysis of urinary protein, urinary enzyme activities. and blood urea nitrogen in the rats given the TOB-LMOX mixture for 15 days showed that the reaction mixture did not produce cytotoxicity leading to renal dysfunction (data not shown). It is considered that in the rats given the reaction mixture, the greater reduction in renal TOB level results from the greater degree of drug interaction induced by preincubation for $3 \mathrm{hr}$ at $37^{\circ} \mathrm{C}$. Thus, this result suggests that the interaction of TOB and $L M O X$ is involved in the suppression by LMOX of intrarenal TOB level.

In the second set of experiments, the administration of LMOX at 0.5 or $1.5 \mathrm{hr}$ before TOB injection or simultaneous injection of LMOX significantly suppressed the intrarenal accumulation of TOB, whereas no significant reductions in renal TOB level were observed in the rats given LMOX at $5 \mathrm{hr}$ before or 1.5 or $5 \mathrm{hr}$ after TOB injection (Fig. 2). To explain the changes induced by LMOX in the renal TOB concentration, we determined the urinary excretion patterns of both drugs. The present study showed that the urinary excretion pattern of TOB almost overlapped with that of LMOX in the rats given both drugs simultaneously (Fig. 3C). We have already demonstrated that TOB directly interacts with $\operatorname{LMOX}(7)$, and TOB (12) and LMOX (13) have been shown to be excreted into urine through the basement membrane of renal glomeruli as a common excretion pathway in rats. Thus, on the basis of these observations, the alterations in renal TOB level induced by LMOX may be explained by the difference in the degree of interaction between TOB and LMOX in vivo, as follows: in the rats given both drugs concomitantly or LMOX at 0.5 or $1.5 \mathrm{hr}$ before TOB injection, reduced renal TOB levels may result from a greater degree of interaction between both drugs, as suggested by the urinary drug excretion patterns. However, in the cases of treatment with LMOX at $5 \mathrm{hr}$ before or after TOB injection. less molecular interaction between TOB and 


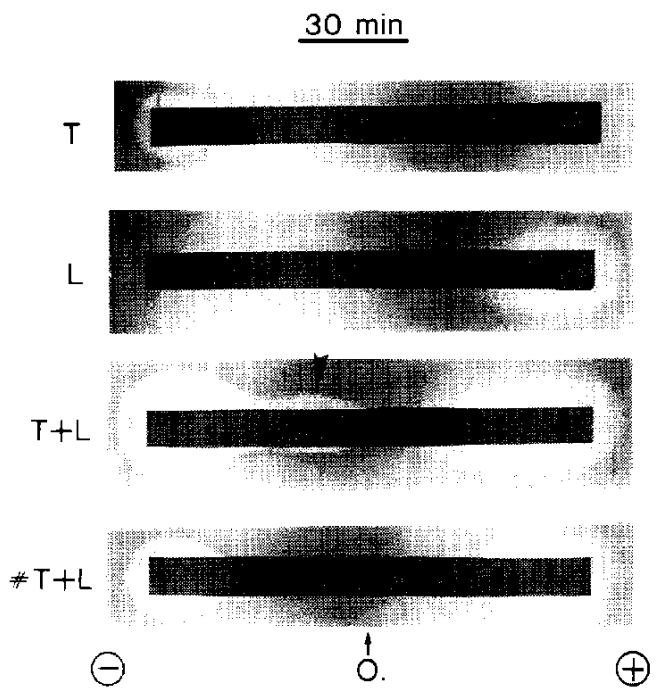

$\underline{90 \mathrm{~min}}$

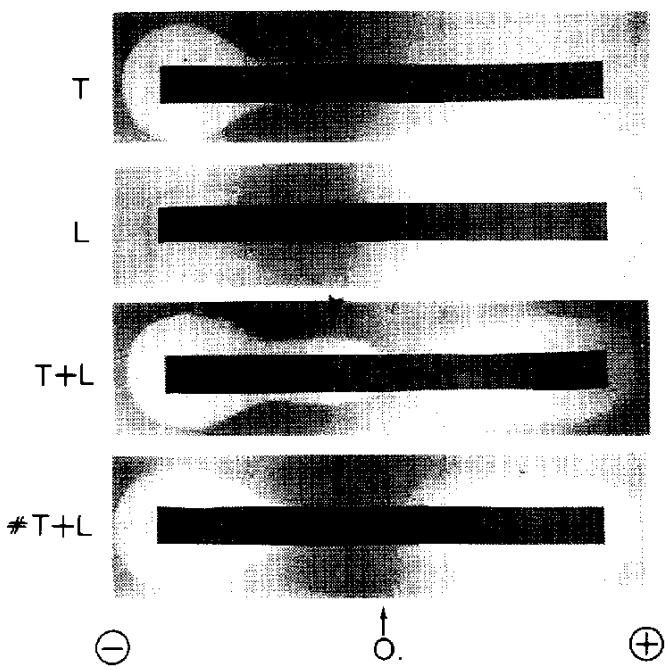

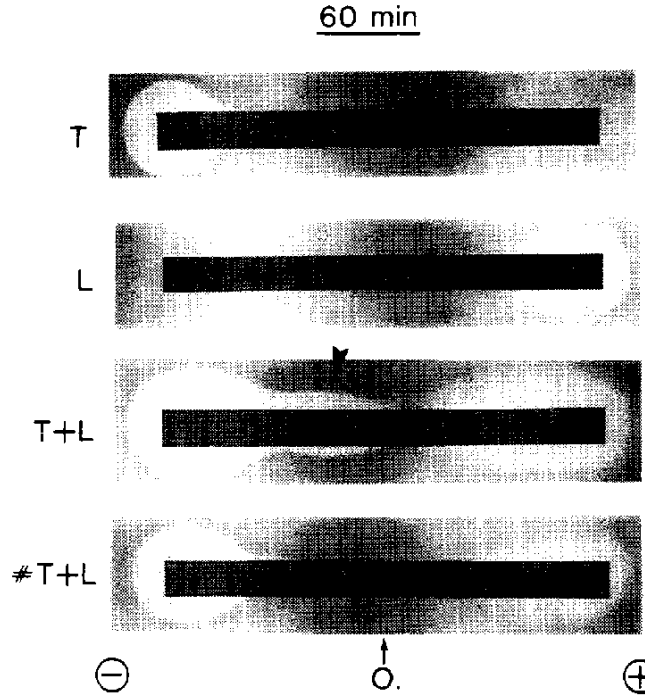

$120 \mathrm{~min}$
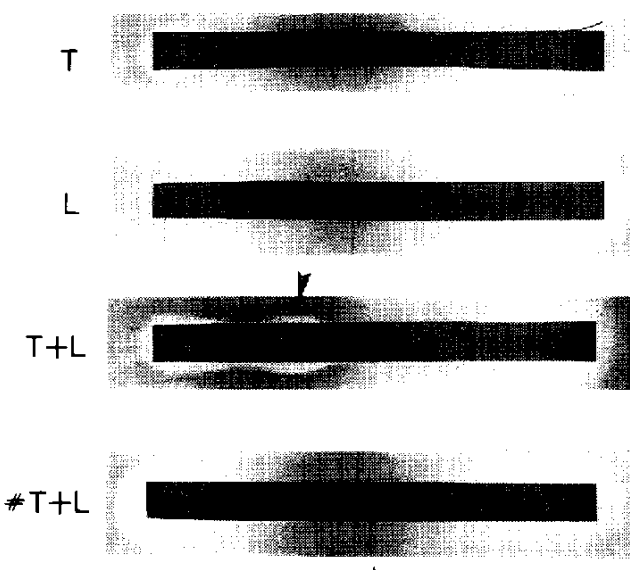

1

$\oplus$

Fig. 5. Detection of the complex of TOB and LMOX in the urine of the rats given both drugs simultaneously. Urine samples were analyzed by the method described in Materials and Methods. O.: origin: T: TOB alone; L: LMOX alone; $T+L: T O B$ and $L M O X ; ~ I T+L: T O B$ and $L M O X$ (each urine sample containing TOB or LMOX was separately spotted at the origin). Arrow heads represent the complex of both drugs.

LMOX, which can be assumed from a combination of each urinary excretion pattern of both drugs (Fig. 3, A and B), may account for the inability of LMOX to suppress the renal TOB level. Additionally, the failure of LMOX injected at $1.5 \mathrm{hr}$ after TOB injection to yield a significant reduction in renal TOB level may be due to a rapid increase in TOB level. which reached to a concentration of about $200 \mu \mathrm{g} / \mathrm{g}$ tissue wet weight before the LMOX injection (Table 1 ).

Finally, we attempted to detect the complex of TOB and LMOX to verify the above speculation. In fact, in this study, we have 
A
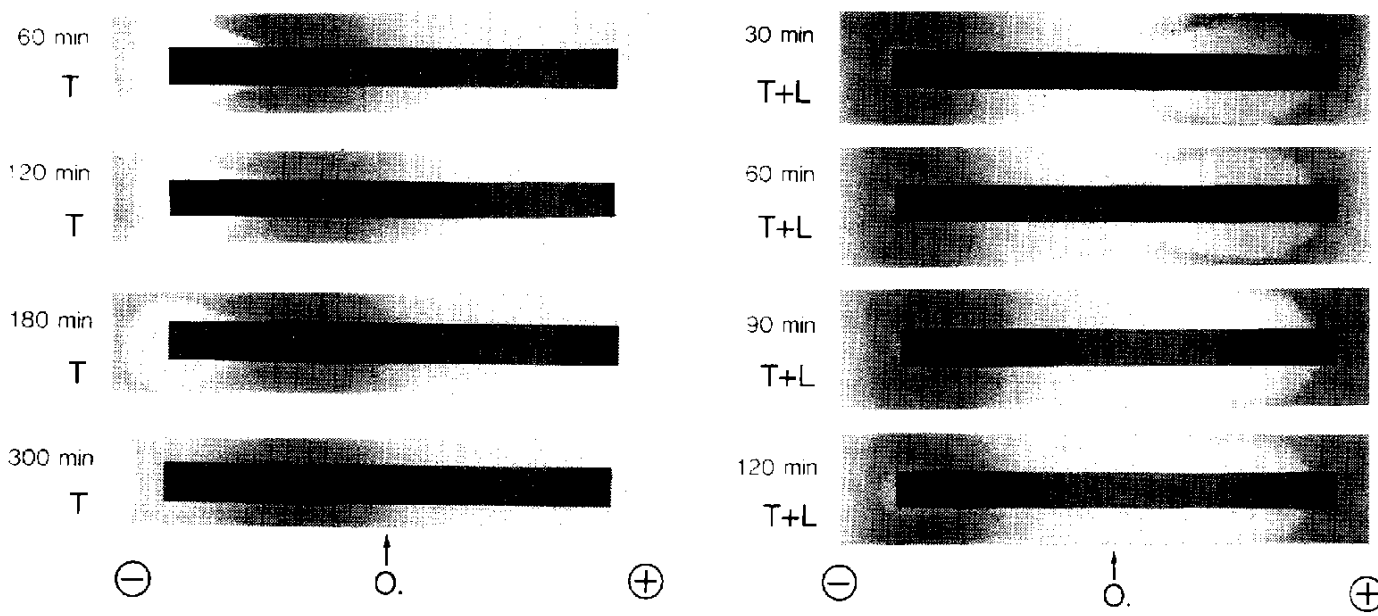

B
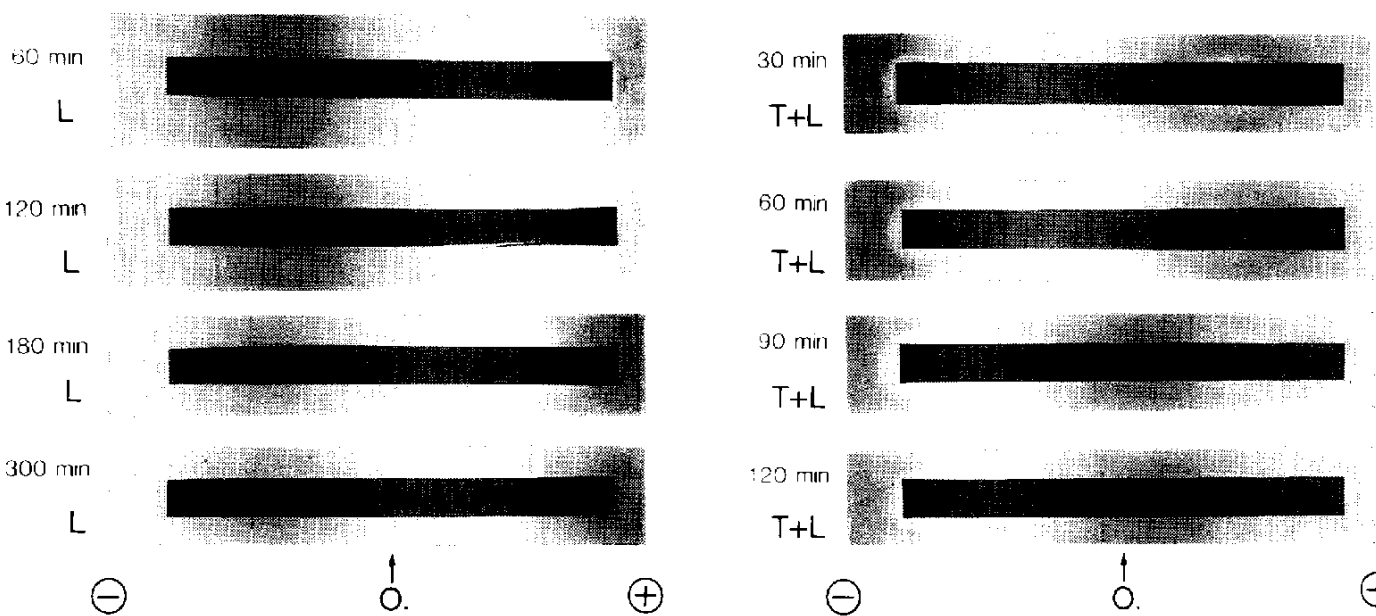

Fig. 6. Detection of the complex of TOB and LMOX in the urine of the rats given LMOX at 5 hr before (B) and after $(A)$ TOB injection. Urine samples were analyzed by the method described in Materials and Methods. O.: origin: T: TOB: L: LMOX.

found that a complex of TOB and LMOX exists in the urine of the rats given both drugs simultaneously, whereas the urine of the rats injected with LMOX at $5 \mathrm{hr}$ before or after the TOB injection shows no manifestation of a complex of the drugs (Figs. 5 and 6 ). These results indicate the possibility that TOB interacts with $L M O X$ in vivo and support our hypothesis that the interaction of TOB and LMOX is involved in the suppressive action of LMOX on intrarena! TOB level.
Various speculations can be made about the site for the interaction of TOB and LMOX. First, although cephalosporin antibiotics such as cephaloridine and cephalexin have been shown to be transported into renal proximal tubular cells across basolateral membranes (14), there is no uptake of LMOX from the antiluminal side of the tubular cells (13), and no accumulation of LMOX in rat tissues is observed by whole body autoradiography (15). Thus, it is unlikely that LMOX induces 
the reduction in renal TOB level inside of the tubular cells. Second, we have found that the pharmacokinetics of TOB in the blood is not changed by combination with LMOX (1), and we could not detect a complex of both compounds in the blood by the method used in this study (data not shown). LMOX has been reported to highly bind to serum albumin (13). suggesting that there is only a small amount of free LMOX which is able to interact with TOB in the blood. Thus, the blood does not seem to be a predominant site for the formation of a complex of TOB and LMOX. Third, both drugs administered subcutaneously are excreted from glomeruli and then are concentrated in tubular lumens. This concentration process of drugs, which increases the degree of access and collision between the compounds, would increase the degree of molecular interaction of both drugs in the tubular lumen. Thus, the renal tubular lumen may be an appropriate site for the drug interaction. However, because in the present study, we can not completely exclude the possibility that the interaction between TOB and LMOX is induced within the catheter inserted into the ureter, a further study using the micropuncture technique (16), which enables the collection of urine from renal proximal and distal tubular lumens, may be needed to detect a complex of TOB and LMOX in the tubular lumens.

It has been reported that some cephalosporins (CEP) including cephalothin protect against $A G$-induced nephrotoxicity in rats and that the protection is due to the suppression by CEP of renal accumulation of AGs (17-19). The mechanism of the suppressive action of CEP, however, remains unknown. Because CEP such as cephalothin have molecular structures similar to that of LMOX, the documentation of the TOB-LMOX complex in our study may raise the possibility that the molecular interaction of AGs and CEP is, in part, involved in the reduction by CEP of intrarenal AGs level.

In conclusion, the results of the present study indicate that the molecular interaction of TOB with LMOX is associated with the suppression by LMOX of the intrarenal level of TOB and provide support for our hypothesis regarding the mechanism of the suppressive action of LMOX on intrarenal TOB concentration.

Acknowledgment: We thank Dr. H. Miwa of Shionogi Research Laboratory. Shionogi Co., Ltd.. for helpful advice in establishing the microbiological procedure with Pseudomonas aeruginosa SR-24. We also thank Shionogi Ca., Ltd. for supplying TOB. LMOX and Pseudomonas aeruginosa SR-24.

\section{References}

1 Kojima, R., Ito, M. and Suzuki, Y.: Studies on the nephrotoxicity of aminoglycoside antibiotics and protection from these effects (3). Protective effect of latamoxef against tobramycin nephrotoxicity and its protective mechanism. Japan. J. Pharmacol. 42, 397-404 (1986)

2 Sastrasinh, M., Knauss, T., Weinberg, M.J. and Humes, D.H.: Identification of the aminoglycoside binding site in rat renal brush border membranes. J. Pharmacol. Exp. Ther. 222, 350-358 (1982)

3 Just, M. and Habermann, E.: The renal handling of polybasic drugs. 2. In vitro studies with brush border and lysosomal preparations. Naunyn Schmiedebergs Arch. Pharmacol. 300, 67-76 (1977)

4 Langford, P.R., Harpur, E.S., Kayes, J.B. and Gonda, I.: Studies of a potential in vitro test for estimation of toxicity of aminoglycoside antibiotics and polyamines. J. Antibiot. 35, $1387-$ 1393 (1982)

5 Wang, B.M., Weiner, N.D., Ganesan, M.G. and Schacht, J.: Interaction of calcium and neomycin with anionic phospholipid-lecithin liposomes. A differential scanning calorimetry study. Biochem. Pharmacol. 33, 3787-3791 (1984)

6 Chung, L., Kaloyanides, G., McDaniel, R., McLaughlin, A. and McLaughlin, S.: Interaction of gentamicin and spermine with bilayer membrane containing negatively charged phospholipids. Biochemistry 24, 442-452 (1985)

7 Kojima, R., Ito, M. and Suzuki, Y.: Studies on the nephrotoxicity of aminoglycoside antibiotics and protection from these effects (5). Interaction of tobramycin with latamoxef in vitro. J. Pharmacobiodyn. 11, 9-17 (1988)

8 Kojima, R., Ito, M. and Suzuki, Y.: Studies on the nephrotoxicity of aminoglycoside antibiotics and protection from these effects (7): Effect of latamoxef on binding of tobramycin to brush border membranes isolated from rat kidney cortex. Japan. J. Pharmacol. 51, 465-473 (1989)

9 Burd, J.F., Wong, R.C., Feerey, J.E., Carrico, R.J. and Boguslaski, R.C.: Homogeneous reactantlabeled fluorescent immunoassay for therapeutic 
drugs exemplified by gentamicin determination in human serum. Clin. Chem. 23, 1402-1408 (1977)

10 Stitzer, S.O. and Martinez-Maldonado, M: Clearance methods in the rat. in Methods in Pharmacology, Vol. 4B, Renal Pharmacology. Edited by Martinez-Maldonado, M., p. 23-40, Plenum Press, New York (1978)

11 Cojocel, C., Dociu, N., Ceacmacudis, E. and Baumann, K.: Nephrotoxic effects of aminoglycoside treatment on renal protein reabsorption and accumulation. Nephron 37, 113-119 (1984)

12 Regamey, C., Gordon, C.R. and Kirby, M.M.W.: Comparative pharmacokinetics of tobramycin and gentamicin. Clin. Pharmacol. Ther. 14. 396-403 (1973)

13 Yoshida, T., Kimura, Y. and Tochino, T.: Pharmacokinetics of 6059-S in experimantal animals. Chemotherapy 28, (s-7), 94-206 (1980) (Abs. in English)

14 Hori, R., Ishikawa, Y., Takano, M., Okano, T., Kitazawa, $S$. and Inui, K: The interaction of cephalosporin antibiotics with renal cortex of rats: Accumulation to cortical slices and binding to purified plasma membranes. Biochem. Phar- macol. 31, 2267-2272 (1982)

15 Sugeno, K., Okabe, H., Tanaka, H. and Norikura, R.: Disposition of $6059-\mathrm{S}$ in rats, dogs and monkeys. Chemotherapy 28 (s-7), 207-235 (1980) (Abs. in English)

16 Lang, F., Greger, R., Lechene, C. and Knox, G.F.: Micropuncture techniques. In Methods in Pharmacology, Vol. 4B, Renal Pharmacology, Edited by Martinez-Maldonado. M., p. 75-103. Flenum Press, New York (1978)

17 Dellinger, P., Murphy, T., Barza, M., Pinn, V. and Weinstein, L.: Effect of cephalathin on renal cortical concentrations of gentamicin in rats. Antimicrob. Agents Chemother. 9, 587-588 (1976)

18 Barza, M., Pinn, V., Tanguay, P. and Murray, T.: Nephrotoxicity of newer cephalosporins and aminoglycosides alone and in combination in a rat model. J. Antimicrob. Chemother. 4, 59-68 (1978)

19 Furuhama, K. and Onodera, T.: The influence of cephem antibiotics on gentamicin nephrotoxicity in normal, acidotic, dehydrated, and unilaterally nephrectomized rats. Toxicol. Appl. Pharmacol. 86, $430-436$ (1986) 